\title{
Fitness Landscape and Tax Planning: NK Model for Fiscal Federalism
}

\author{
Marisa Faggini ${ }^{1} \&$ Anna Parziale ${ }^{2}$ \\ ${ }^{1}$ Dipartimento di Scienze Economiche e Statistiche, University of Salerno, Italy \\ ${ }^{2}$ Dipartimento di Scienze Giuridiche, University of Salerno, Italy \\ Correspondence: Marisa Faggini, Dipartimento di Scienze Economiche e Statistiche, University of Salerno, Via \\ Giovanni Paolo II, 132- 84084 Fisciano (Salerno), Italy. E-mail: mfaggini@unisa.it
}

Received: April 18, 2017

Accepted: May 17, 2017

Online Published: May 25, 2017

doi:10.5539/ijef.v9n7p14

URL: https://doi.org/10.5539/ijef.v9n7p14

\begin{abstract}
This paper rises from the idea to highlight how traditional models of Fiscal Federalism are not be able to capture adequately the behavioral dynamics of economic systems. We stress the innovative aspects of complexity theory and the premises on which to base the analysis of Fiscal Federalism in this perspective. For this purpose, we consider Fiscal Federalism as a network of economic relationships between different complex adaptive and co-evolving systems, the jurisdictions, linked by strong interdependencies. We will proceed to model a landscape in which co-evolving jurisdictions have to find the optimal path to organize the local tax planning and to optimize their local economy.
\end{abstract}

Keywords: fiscal decentralization, coevolution, fitness landscape, tax planning

\section{Introduction}

Federalism, both in theory and in reality, is a commonly used label to identify a wide range of political and institutional models characterized by the union of a functional and structural multiplicity of local authorities, variously named, but all have, more or less extensive powers of self-government. Federalism, in this sense, means many things, among them often different and sometimes seemingly antithetical and, indeed, no model of Federalism, actually exists, equal to another.

Also from the theoretical point of view there are significant differences in the approach to this issue. Buchanan (1965) for example, prefers a reading near to political philosophy and using the analogy between clubs and local government, he proposed to explain the behavior of local governments in order to determine the optimal level of both size and activity. Musgrave (1959), however, considers Federalism primarily in terms of the theory of public finance, suggesting that the three are the functions assigned to the public sector: macroeconomic stabilization, income redistribution and resource allocation. The first two have to be the exclusive prerogative of the central government, while the allocative efficiency of the decentralized governments. It follows an "easy" translation of these assumptions in the theory of an appropriate system of Fiscal Federalism, which is to maximize the satisfaction of individual preferences over public goods and services through the decentralization of public expenditure and revenue decisions.

However, the pursuit of policies of public intervention, as any decision to maximize an objective function, in this case, the welfare of the community, through efficient public spending, cannot ignore the constraints of available resources. It would derive, otherwise, a set of distortions in the evaluation of policies that can affect in the long time the goodness of public intervention itself.

In fact, it is not possible to consider optimal a choice that identifies the benefits of the intervention, but not the costs related to it and especially without taking into account the effects that the marked differences that characterize the reality of the individual territoriality, could have on the sustainability of federal structure in the active pursuit of fiscal policies. We must therefore make suitable choices for a complex and complicated reality and discard others that, although theoretically valid, result inconvenient when put in a heterogeneous environment.

Traditional economic models of Fiscal Federalism do not take into account these observations but they simplify the described reality. If, however, it is true that the cognitive process is at the same time a simplification process, (because it do not perceive the reality of things but its phenomenology), this does not mean it have to dismantle 
too the layer of complexity that surrounds the nature of things.

This is the basis of analysis of Complexity Theory. Complex is each phenomenon not completely framed in a linear, deterministic and predictable context. It is different from what, until now, the science has formalized by following the principles of separation, reduction and abstraction. These principles, imposed by Cartesian paradigm of simplification have created a separation between reality and its formal representation. The Complexity Theory studies the phenomena not more by simplifying, linearizing and dividing them, but observing the relevance of inter-relationships among the components of systems - as well as their relationships with the environment and vice versa - in determining collective behaviors.

In this sense economics is a complex system but also a co-evolutionary system. The co-evolution describes the evolution of two or more agents that interact closely with another one and with the environment, reciprocally affecting each other's evolution. Further, because these agents are part of their environment, when they change, they change also their environment, and as it has changed they need to change again, and so it goes on as a continuous process. Each agent continually has to reorganize itself in order to seek a sufficient level of performance (fitness) to survive. In other words within this changing landscape, agents have to continually seek optimal positions and each strategic choice of a system leads to position changes of the others in unpredictable and unplanned ways. However, from these mass interactions regularities emerge and start to form a pattern that feeds back on the system and informs the interactions of the agents.

From a mechanistic and linear vision, inspired by the Newtonian principles, they are now moving towards a complex approach where the whole is more than the sum of parts. The variables that measure the macroscopic state of a system, influenced by microscopic forces, can manifest linear or alternatively non-linear dynamics, in this last case, coherent or purely chaotic dynamics.

The paper is structured as follows: after the introduction in section 2 we propose a brief review of the latest literature on Fiscal Federalism. Section 3 highlights the weakness of traditional models of Fiscal Federalism to capture adequately the real behavioral dynamics of economic systems. We stress the innovative aspects of complexity theory and the premises to analyze Fiscal decentralization in that perspective. We focus the attention to concepts such as interactions between agents, non-linearity and co-evolution. In section 4 we briefly describe the fitness landscape and the NK model of Kauffman (1993) to analyze the evolutionary dynamics of complex systems and we stress the relevance of evolutionary theory in economic studies. In section 5 we explain how patching theory may be offer solution for decentralized political decision-making structures. Then we describe a landscape model within jurisdictions, complex systems of small size, move on to find the optimal path to optimize their local economic organization. Finally, the properties of Kauffman's random exploration with a dynamic that reduces the randomness by introducing small constraints are compared. The work concludes with some considerations.

\section{The Weakness of Traditional Economic Models}

Traditionally the theory of fiscal decentralization (Note 1) is focused on three essential aspects: i) the sharing of functions between the different levels of government, particularly at four levels to supply of public goods and services; ii) macroeconomic stabilization as well as taxation and redistribution of income; iii) the use of the instruments of fiscal policy (particularly issues associated with taxation and inter-governmental transfers). Recently, the identification of welfare gains resulting from fiscal decentralization is considered. (Spahn, 2006).

Dominant modeling of economic theory on Federalism until the 1980 (Buchanan, 1965; Musgrave, 1959; Oates, 1972; Tiebout, 1956), shares a common approach: the simplification and abstraction of the assumptions of generally linear and static models, able to offer unique and deterministic solution. The Oates'model suggests, for example, the absence of "spillover effects" and economies of scale, constant production costs, but also uniformity of preferences within local government jurisdictions and the heterogeneity of preferences among local jurisdictions. Oates achieves, in fact, "not ambiguous results", just because he departs from these assumptions. Moreover, the respect of the "correspondence principle" is difficult not only cause of the difficult determination of the territorial scale of a single good, but also because, generally, different public goods will have different optimum dimensional areas. Oates's decentralization theorem has a clear rationale.

Welfare is maximized when specific public goods are provided by local governments, whose jurisdiction corresponds to the subset of the national population for which the demand for specific public goods and services is homogeneous. Oates's assumptions on uniform provision of public goods by national governments do not reflect reality in any strict sense. The homogeneity of preferences characterizes also Buchanan's model, to which the assumptions of the existence of preferences revelation mechanism and a population with the same income had added. Starting from the heterogeneity of preferences within the jurisdiction Tiebout assumed that the 
individuals could move freely among the different jurisdictions offering different baskets of goods (government services) at a variety of prices (tax rates). Because individuals have different personal valuations on these services and different ability to pay the attendant taxes, they will move from one local community to another until they find the best mix of services and taxes that maximizes their utility. With enough variety among the jurisdictional offerings, each community will end up with people having identical preferences.

Through this choice process, an equilibrium provision of local public goods in accord with the tastes of individuals will be determined. While the model has the advantage of solving two major problems with government provision of public goods: preference revelation and preference aggregation, however it relies on a very restricted set of assumptions. Perfect mobility, perfect knowledge of the differences among the various local governments in terms of taxes to be paid and services to be used; large number of jurisdictions, limited relevance of spill-over effects, constant-cost of services production allow to obtain an efficient provision of public goods.

The result is what we call the first generation theory of fiscal decentralization. The final stream of the first generation theory derives from the public choice literature, which reaches back into intellectual history for its motivation. Under this approach (Brennan et al., 1981). Central governments do not maximize social welfare and act like monopolists (or leviathans) in order to increase their control over the economy's resources. Oates and Tiebout offer a theoretical framework in which fiscal decentralization can guarantee an efficient provision of public goods simply because local preferences are satisfied better than the centralization. Both previous approaches assume a benevolent government, but the Leviathan hypothesis is based on the opposite assumption whereby decentralization is a means to reduce government size in order to stem its inefficient behavior. The important contribution of first generation theory is that it reveals that, generally, efficient levels of publicly provided outputs are achieved more through multi-levels systems of government than through a unitary system of government. Welfare benefits from decentralization are likely to be greatest when there is a diversity of preferences for impure local public goods. However, the limits of these findings, due to the restrictive assumptions used to derive the decentralization theorem, cannot be overlooked.

In the last twenty years, the classical approach evolved together with the theory of fiscal federalism in what is sometimes called the second-generation theory of fiscal federalism (Oates, 2008). The emerging second generation theory has been characterized in terms of two motivating issues: incentives and knowledge incentives are required for subnational government to do a better job to avoid outward migration of people and firms; and knowledge of local preferences and tastes is crucial to achieve economic efficiency when local public goods and services are provided by subnational government. Both motivations have contributed to an increased economic efficiency. In this new literature the effect of fiscal decentralization has been modeled to embody the political process and the possibility of asymmetric information across political agents. Differently from the classical approach governments are assumed to maximize their own objective function that does not imply the maximization of social welfare. This new literature reconsiders the decentralization theorem in a political economy contest. Here the main argument in favor of decentralization hinges on the inefficient outcome of the centralized decision-making process rather than on the trade-off between preference matching and externalities typical of the original version of the Oates' theorem. On the other side studies the trade-off between centralized and decentralized provision in principal agent models of electoral accountability.

The contributions of the second generation theory are mainly drawn from the economics of transaction cost, incomplete contracts and principal agent perspectives. Leading studies that have been classed as parts in the emerging second generation theory, are associated with Weingast (1995), Seabright (1996), Besley and Coate (2003) Lockwood (2007). Weingast (1995) introduced the notion of "market preserving federalism" to investigate how competing jurisdictions create incentives for credible commitment and lower transaction costs. In contrast, Seabright (1996) introduced the notion of 'incomplete contract' to the analysis of fiscal federalism. The author presents elections as incomplete contracts in which some information, in the 'contract', is unverifiable. Political accountability can be an organizational motivation for decentralization. In contrast, centralization could be more preferred when the mechanisms associated with incomplete contract provide greater scope for policy cooperation between different levels of government to internalize inter-jurisdictional fiscal externalities. As a consequence, the decision on preferred mechanism depends on the relative magnitude between benefits from internalization of inter-jurisdictional fiscal externality and costs arising due to a reduced accountability under fiscal centralization. The starting point for Besley and Coate (2003) and Lockwood (2007) and is Oates's decentralization theorem, but these authors correctly pointed out that goods and services provided by the national government are not necessarily homogeneous, as Oates had originally assumed.

Once it is recognized that national provision of public goods and services is possible on a differential basis between regions, a different fiscal framework is needed from that developed by Oates. The generally accepted 
view now is that the presence of the second generation theory of fiscal decentralization classifies, rather than contradicts, the validity of the first generation, including the decentralization theorem: Although the models under the second generation theory umbrella differ in fundamental ways from the first generation theory, many of them produce a trade-off between centralization and decentralization that is in a somewhat similar spirit to their earlier counterparts (Oates 2005, 2008).

\section{The Complexity Theory Perspectives for Analyzing Fiscal Federalism}

Federalism is also about the recognition that all levels of government and of political struggle interact with one another. The sharp distinction between domestic and international politics and the analysis of one aspect in complete isolation from the others cannot bring a fully satisfactory comprehension of either sphere. If politics and society were as simple as many hard-nosed realists assume, the researcher's job would be easier. Unfortunately, the world is complex, plural and nuanced. This requires an open-minded attitude to other disciplines and perspectives to try to cope with this complexity and grasp the fundamental linkages of the interdependence figurations which characterize and influence individual and group behaviors at different levels of analysis.

Mainly, theoretical structure and, consequently, the application of all the economic models, depend heavily on the basic assumptions, which represent their cornerstone but at the same time their Achilles' heel.

In fact these models are derived, in large part, by the translation in a simplified form of the insights of researchers on the reality, paying a hefty price due to the limits of this procedure, which is common to all sciences. It concerns the way in which the simplification is made and, above all, the level of simplification up to which we must or can be pushed without causing the loss of important and explicative information. Therefore it is not questioned whether the simplification must be made or not. The formal models that meet general approval are those which, although with some degree of abstraction, maintain a strong relationship with the represented phenomenon. More controversial, however, is the validity of those models that substantially deviate from what they are trying to approximate, in spite of their apparent ability to synthesize better than less formalized models.

There are many conditions in which opposite effects are algebraically added, neutralizing each other. Generally, to be able to catch them, it used an assumption that has the idea of an average that considerably summarizes the description. If, however, it disclaims investigating the underlying interactions it loses much of the informational value of the result and it accepts the risk that, increasing the level of generality, the model will prove totally unfounded.

To overcome these limits and the growing interest in the dynamics of evolutionary systems, researchers from different disciplines (physics, biology, economics) have started on the one hand to test the goodness of traditional theories and models, proved, in fact, often unable to adequately capture the behavioral dynamics of systems, and on the other hand to explain the new principles that would provide a justification for such inadequacy, forming the foundation for the construction of a new interdisciplinary approach: the Complexity Theory (Bertuglia \& Vaio, 2006; Colander et al., 2004; Arthur et al., 1997).

From a mechanistic and linear view of where the entire is always equal to the sum of the parts we are moving to a non-linear, complex view where "the entire is more than the sum of its parts". The linear view represents only one of many states in which a system can passes through: chaos and order coexist and the key to understanding all is the degree of interaction between the various elements that compose the system.

What is interesting is the analysis of the behavior of "system-model" located in "environment-model" in order to understand how, through co-evolution the system adapts to the environment and vice versa from time to time resulting different configurations (Oliver \& Roos, 1999; Stacey, 1995, 2003).

Systems and the environment have been studied often in the unique perspective, which had as its main, while not only, knowledge objective to determine the effects ex post generated from operating in the contexts of the subjects, without taking into consideration the "reciprocal" nature of the phenomena and, therefore, never resorting to the identification of a working scheme of their interaction in time and space by adopting i.e. a co-evolutionary approach.

Speaking of co-evolution, then, implies the need to have a dual and contextual perspective of investigation, the perspective of systems and of the environment, in which the economic and also the anthropological variable are strongly represented and interdependent.

It is a contextualized system in time and space the features of which are the fundamental variability of the environment (landscape) and the ability to use the environment as a source of competitive advantages depend on fitness levels. The theory of fitness has been proposed in evolutionary biology to represent the relationship 
between the number of genotypes of a certain class found in the present generation and the number of the same class of genes identified in the previous generation (Wright, 1932).

Economic science has translated the concept of fitness in an evolutionary theory according to which heterogeneous organizations are selected on the base of their ability to develop different levels of fitness, represented by fitness landscape, with the environment wherein they operate. (Nelson \& Winter, 1983).

Therefore the study of the characteristics of the system-environment relationship must take into account that, because of the interaction, any evolutionary change of a system can lead to evolutionary changes in another, and that, under the co-evolution, the improvements for a system will provide competitive advantages for another allowing finding much of the available resources. In this context, the fitness increase of a system is due to decreased fitness of another system. The only possible solution for a system involved in this competition, is to adapt continuously as fast as in order to maintain its fitness level compared to that of other economic systems and alternately change its configuration.

Since the environment in which systems operate continuously changing as a result of co-evolution between them and the environment, the purpose of each system is to optimize their level of fitness as an expression of the attitude and the ability to survive via typical adaptation mechanisms of natural selection.

This is important since the majority of economic activity involves the integration and coordination of interdependent resources. Some of these interdependencies are that an element of the system needs other element to perform its function, or at least it can perform well its function if the other element is also present. It is therefore helpful to think of an economic system (enterprise, firm, production system, a jurisdiction) as a network of connected elements by a dense and complex links of interdependencies.

We can say the same to frame the Fiscal Decentralization in this framework of analysis. We consider the public sector as a big complex adaptive system in which different forces, hardly compatible, act with a multitude of human beings, with variables moods and continuous changes in political and economic scenarios. The fiscal decentralization as a prerequisite for organizing the entire fiscal structure led to the creation of local jurisdictions with fiscal autonomy.

The jurisdictions are economic systems at many dimensions characterized by complexity at different hierarchical levels. In this sense they are complex systems characterized by the connections between different levels and sizes through communications network. Economic agents are the nodes of the network, which produce knowledge by processing the information (Barabasi, 2003).

The jurisdictions play a very important role in the development of a country's competitiveness for economic development and that is why it stresses the need to develop an integrated and coordinated strategy that in a necessity bottom up logic, shifting the emphasis from static to dynamic optimization, is based on the use and development of new research tools such as genetic algorithms, exploration models, and simulations to analyze the potential long-term consequences of fiscal choices, their adaptability and robustness through the change of scenery.

\section{NK Model and Economic Complex Systems}

To analyze the effects of interdependences on complexity system, we describe the theoretical core of fitness landscapes starting from and the NK model formalized by Stuart Kauffman (1995). This model consists in the search of optimization for problems characterized by a large number of variables in conflict with each other.

We consider a system composed of $\mathrm{N}$ elements that can have different states ( 0 and 1$)$. These elements may have also different degrees of interdependence. These interdependencies are considered if they were determined randomly. The aim is to check in detail is the "degree of interdependence" in the system, i.e., the average number of other elements with which each element is interdependent.

$\mathrm{K}$, whose values are between 0 and $\mathrm{N}-1$, denote the measure of interdependence. We denote whit system configuration each possible combination of states of individual element and whit fitness the measure of the system performance. Each possible configuration of the elements of the system will have its own degree of fitness, more or less dependent on complementarity (Note 2) and the greater or lesser effects of conflicts between systems. From the set of fitness values associated with different configurations of the system arises a "surface" called fitness landscape.

NK model can be considered as composed of two distinct components: a specific problem and a searching algorithm in the space of possible solutions. As we have said, the problem is a set of possible solutions represented as binary strings, each associated with a value of fitness, which is the pay-off of that solution. The 
NK model analyzes the evolution of a single string, which represents the state (or configuration) of a system, and it is important, although preliminary, for the construction of more elaborate models, in order to suggest possible avenues for self-organization in situations characterized by co-evolution.

The relevance of evolutionary theory and the NK model in the study of economic organizations has been detected several times in the literature Westhoff et al. (1996), Levinthal (1997), Pagano (2013), Frenken (2001, 2005, 2006), Frenken and Valente (2004). Gerrits et al. (2014), Barkley Rosser Jr (1999, 2012). In Frenken (2001) and Frenken and Valente (2004) the authors, following Kaufmann's NK model theory, suggested a formalisation of network organisations in searching a complex fitness landscape of technological artefacts and innovations characterised by conflicting constraints due to interdependencies between its constituting elements. The central question is what modes of organization can be distinguished in designing a complex system and how can their performance of search activity in complex systems be compared. They distinguished three types of organizational modes a centralized organization, a decentralized organization and a network organization. These studies however, neglect the problem of co-evolution, which instead underpins this work.

Kauffman (1993) restricted his analysis of complex systems to particular types of architectures expressed by one parameter $\mathrm{K}$, which stands for the number of elements. This parameter can be considered as an indicator of a system's complexity, with $\mathrm{K}=0$ being the least complex and $\mathrm{K}=\mathrm{N}-1$ the most complex architecture.

In our case, a low value of $\mathrm{K}$ highlights little interaction among policy choices of different jurisdictions, so the fitness landscape is smooth or highly correlated; therefore, a change in one policy has little impact on the fitness contribution of other jurisdictional choices. In contrast, a high value of $\mathrm{K}$ implies that a change in one jurisdiction policy has a large impact on the fitness contribution of other jurisdictional choices. Therefore, given an initial setting of incremental change in the vector of $\mathrm{N}$, policy jurisdictions may substantially change the overall payoff level. As a result, the fitness landscape becomes less correlated, or equivalently, more rugged, with a higher $\mathrm{K}$ value. When there are significant interaction effects among policy variables, there may be a number of local peaks.

Moreover, if Kauffman's work exploits to a large extend the simulation of large complex systems, we pursue as far as possible an analytical approach to the presentation of the main qualitative properties of the models.

To clarify our intent, we consider a process of co-evolution between jurisdictions induced, for example, by the need to reorganize their economic system as a result of tax reform.

The reorganization of tax planning and the opportunities for economic growth, given scarce resources, causes a competition between jurisdictions. This is a phase of substantial uncertainty, caused by the fact that new opportunities are still ill-defined and can evolve rapidly. This situation drives to new dominant solutions as a result of an extensive process of co-evolution influenced by interdependence.

If the effects of interdependence between jurisdictions are strong enough, the results of co-evolution in each jurisdiction are disturbed by the systematic deformation induced by simultaneous evolution of the fitness landscape in the other jurisdictions.

In this scenario, the constraints of interdependence plays a selective role because they affect the likelihood that the systems are well adjusted. This occurs because the interdependence constraints, by limiting the set of advantageous movements in the space of representation of the possible solutions, they increase the probability of evolution towards a stable configuration (despite the fact that the configuration may not be optimal ex post). In this way, the interdependence constraints help to reduce uncertainty and disorder in a system, considered as a set of evolving complex systems.

If the systems have a sufficiently large number of $\mathrm{N}$ elements, there is a trade-off between the probability that a process coevolves towards a stable local peak and the average fitness of a peak. So, it turns out that systems with an intermediate degree of interaction have a selective advantage against competitors, characterized by very high or low complementarity constraints. These properties are always true, no matter if evolution proceeds by random exploration of such trial and error (Kauffman, 1993; 1995), or by imposing constraints that help to identify optimal choices within a set local choices. Once the systems are simultaneously at peak fitness, co-evolution tends to decrease.

\section{The Patching Theory and the Effects on Sub-System Levels: The Jurisdictions as Patches}

A system moves around its fitness landscape, through various mechanisms: the adaptive walk that estimates the effects of individual changes on the entire system and the patching, (according to Kauffman more efficient), which estimates the effects on sub-system levels. 
Patching theory proposes to divide a complex adaptive system, and then the problems, in several not overlapping parts, the patches. The patches, however, are not independent of each other, or each agent of each patch pays attention only to what happens in its borders, losing sight of the unity of the system and of the problems to solve. It is important to remember that the aim is always the efficiency and the survival of the global system, and then the originated sub-systems from its division constantly have to exchange information and co-evolve together.

Therefore, the patching algorithm searches improvements in the local fitness, inside the patch, rather than global improvements. Instead of adopting changes in the state that have a positive impact on the entire system, it shall state changes that have positive impact on subsets of the system.

Kauffman (1993) argues that this process seems to be particularly suitable to study social systems in which people work in separate groups by creating and not resolving conflicts of different kinds because the individual solutions do not converge towards a single compromise that can properly address all the needs. He highlights that for systems with various types of local autonomy, the analogy with the patches can be a key mechanism for understanding the evolution of economic systems.

It is, therefore, that the theory of fiscal federalism and the patching theory propose to analyze complex economic-financial issues of a complex economic system/ State in the same way by identifying jurisdictions with patches.

Using the patching theory it also addresses the question of possibility, during the adaptive walk, to get into areas of fitness landscape with low efficiency and low fitness value. To avoid such mishaps it should leave the patches individually and freely evolve and auto-organize themselves.

As with other complex adaptive systems, Post and Johnson (1998) assert that "problem of this sort are computationally intractable, incapable of true solution by any known methods [...] legal theory would be enriched $\ldots$ by paying attention to the study of various algorithms derived from the study of "complex adaptive systems' that can successfully operate on problems of this kind" (Post \& Johnson, 1998, p. 1059).

From the complexity theory perspective, there are several kinds of problem solving algorithms, two of which are relevant here. One is a simple trial-and-error method known as the simple adaptive walk. The adaptive walk is an effective algorithm for finding the highest point on the fitness landscape for systems with no interconnections or spillovers between elements. "In systems with substantial spillover effects, however, the algorithm performs progressively less and less well. On these more rugged fitness landscapes, the adaptive walk is increasingly likely to become trapped on local fitness peaks - places on the fitness landscape from which there are no steps leading upwards at all" (Post \& Johnson, 1998, pp. 1075-1076) (Note 3).

For systems with substantial spillover effects there is a different algorithm, called patching, which is a variant of the adaptive walk (Note 4) The patching algorithm is described in depth by Kauffman (1995), and was discovered by Kauffman and his colleagues at the Santa Fe Institute. In a patching algorithm, each element in the system is assigned to a single group of elements, or patch. As an element is "flipped", the fitness of the patch is recalculated. The individual element is permitted to move from one state to another if, but only if, the effect of the move is positive on the aggregate fitness of the members of its patch. Thus, "[t]he patching algorithm seeks local, within-patch improvements in fitness rather than global improvements.... Each patch is allowed to maximize its own fitness, independent of any effects on the fitness of non-members or on the aggregate fitness of the system as a whole" (Post \& Johnson 1998, p. 1078).

Regard to the role of inter-jurisdictional spillover in patched systems, they suggested that the efficiency of dispersed decision-making processes is not a simple inverse function of the magnitude of inter-jurisdictional spillovers; the effective functioning of the patching algorithm not necessary depend by configuring the boundaries between jurisdictions in such a way that all inter-jurisdictional externalities are internalized. The systems with high congruence "appear to be more efficient at finding system-wide fitness peaks than those with more inter-patch spillover" (Post \& Johnson, 1998, p. 1091).

In the systems they examined, "perfectly congruent systems with no inter-group externalities was often less effective at finding system-wide optima than systems with somewhat lower degree of congruence" (Post \& Johnson, 1998, p. 1091).

Their results suggest that search efficiency may decline if congruence is both too high and too low. The systems with an intermediate level of congruence are better at finding higher points on the fitness landscape than systems whit high congruence in which spillovers were perfectly internalized within patches. The same is for systems whit low congruence in which spillovers are only weakly internalized within patches.

Thus, patching is an adaptive walk over a patched system. This similarity by complexity theorists has been 
explicitly recognized with federalism described as a patching algorithm for solving public policy problems. Post and Johnson (1998) provide an exemplary discussion linking the same concept between interjurisdictional relationship, decentralized decision-making process and complexity theory.

As they highlighted the implications of their work for federalism may come as a small surprise to those familiar with theories of competitive federalism.

"Patching may be more than merely a metaphor for decentralized political decision-making structures (though it is that and no less interesting because of it); those structures may, in a sense, be instantiations of the patching algorithm in the political realm. Federalism may "work," in other words, because it is a "patching algorithm," a means for solving public policy problems defined over a most complex "social welfare landscape." As such, an understanding of the factors that determine the effectiveness of the algorithm cannot help but have an impact on our understanding of these political decision-making institutions". (Post \& Johnson, 1998, p. 1090).

Therefore, starting from biology studies, the competitive decentralization could be described as a complex system that is composed of a set of parts of the system, which jointly determine the national welfare. Only if some combinations of system parts are complementary the result can be a high national welfare. Conversely, if the combinations of system parts are incoherent, the result is a lower level of national welfare. The interdependencies between policy jurisdictions indicate that the choice of one strategy cannot be made independently from the choice of others. The existence of interdependencies thus provides a rationale for coordination of search activity at a centralized level. More of these independent actions of system elements can be handled using genetic algorithms to approach search problems (Note 5).

Regarding fiscal federalism, local jurisdictions independently develop themselves and organize their own structure for the collection and spending of financial resources as they see fit. All this, however, within the limits set by national legislation, which must coordinate the process of adaptation of individual geographical areas in order to reach the highest peak of the fitness landscape for the entire State. In this situation the increase in efficiency can be spread with a proper management of externalities, trying to delete the negative ones and encourage positive ones. Dividing a complex system into independent self-optimizing decision-making patches can increase the efficiency of the search for optimal system-wide configurations. In fact, dividing a decision-making policy into subunits may be subject to fewer inefficiencies of information transfer; therefore, local governments and consumers will be more likely to make better (welfare-maximizing) decisions.

Optimization across a fitness landscape involves using optimizing search algorithms not only to control for direction, but also to test the fitness of different system component combinations and adapt to the results continuously. The system's optimization algorithm must be adaptive, moreover, because the systems with which it interacts are evolving in their own searches for the most fit solutions. Complex, adaptive, evolutionary systems incorporate algorithmic decision making tools that allow adaptive long-term fitness optimization through repeated reevaluation of system design.

In this way the algorithm tries to find a single solution to a complex problem by mutating and selecting bit-strings that represent individual solutions to the problem. In fact, the main idea is that if the best solutions are selected in many iterations, the algorithm would converge to a single very powerful solution in the end. However, algorithms often get trapped on a poor solution and several runs often generate different solutions. This outcome has striking similarities to natural evolution whereby the ultimate complex problem is self-replication, which results in greater diversity of species.

In what follows, we show how Kauffman's model can be used to formalize (Note 6) the phenomena mentioned above.

\section{Jurisdictions in a Landscape Model: The Research of Optima}

Let us we assume that the possible levels of public spending of jurisdictions are uniformly distributed in space $K$, where $K$ is obviously the measure of interdependence. To take a systematic relationship between interaction and contributions means that every fitness landscape is drawn from a distribution such that the degree of interaction of an element is correlated with its contribution fitness. A stronger interaction leads to stronger constraint of complementarity.

On this premise, more integrated fitness landscape is even more rugged in the average. Then, as evidenced by Kauffman's results in rugged landscape local optima are more numerous, although their average fitness value may be lower. In addition, routes to the local optima involve fewer steps. These properties can be used to prove that, at every stage of a co-evolutionary process evolving systems on a rugged landscape are more likely to be simultaneously on a peak of landscape, and then to move towards a local optimum. 
For optimal fitness, it is necessary to tune $\mathrm{K}$ depending on coevolution. In the fiscal policy context, the value of coevolution is determined by the extent to which the policies of an opponent directly affect one own policies. Coevolution cannot be controlled, but the value of $\mathrm{K}$ can be modified by adjusting the extent to which some sets of rules cancel out or modify the effects of other rules within the organization.

Coevolution in a landscape model deals with rules. Jurisdiction's tax planning is generally tax rule-following. Public expenditure reflects the implementation of tax rules established at a prior point in time rather than resulting from the novel solution of an optimization problem. Tax rules are not constant - they are changed as a result of catastrophic failure or incrementally — but they change slowly except in unusual situations such as crises.

Low K systems improve their performance very slowly, since rules must be changed one by one without synergistic effects. High $\mathrm{K}$ systems can be changed more rapidly because the change in one tax rule can affect a large number of other rules.

We indicate the level of per capita public expenditure, $a_{i}(t)$ in the jurisdiction $i$ at time $t$. The level of public spending is strictly correlated to the tax planning of the jurisdiction. The information on the level of public spending are coded in a number of binary elements, each of which may have the value 0 or 1 . We can think of the string as a way to encode a specific combination of supply of public goods and services. In each stage of research the number of potentially available combinations tends to grow over time and the length of the string. To emphasize that the suggested approach has little to do with determining size of the problem, it is assumed that the length of all strings is finite and fixed. The efficiency of the chosen level of pre-capita public spending, represented by the fitness value, defines the competitive strength of the jurisdiction vis a vis the other jurisdictions.

There are $\mathrm{G}$ jurisdictions in the country. The level of public spending in the jurisdiction $i(i=1, \ldots, G)$ is a string of $N$ binary elements $\left(x_{i 1}, x_{i 2}, \ldots, x_{i N}\right)$, where each $x_{i j}, j=1, \ldots, N$ can take value 0 or 1 . Then there are $2^{N}$ possible levels of public spending for the Jurisdiction, corresponding to the number of different states in the space $\{0,1\}^{\mathrm{N}}$ that define the set $A_{i}$. We assume for simplicity that at the initial moment there is the same level of public spending in each jurisdiction. The configuration (planning) of the tax jurisdiction is defined by the level of public spending in i. Let $x_{i}$ and $x^{\prime}{ }_{i} \mathrm{~N}$-strings in $A_{i}$. The distance between $x_{i}$ and $x_{i}^{\prime}$ is defined by the number of components having different value with respect to the corresponding components of the neighbor strings (Note 7).

More formally:

$$
d\left(x_{i}, x_{i}^{\prime}\right)=\sum_{j=1}^{N}\left(x_{i j}-x_{i j}^{\prime}\right)^{2}
$$

The neighborhood of $x_{i}$ is the set of strings in $A_{i}$ with distance from $x_{i} \leq 1$ and it is composed of $x_{i}$ and its $N$ neighbors. The fitness function of the jurisdiction is the map $F_{i}: A_{i} \rightarrow \Re$ that associates each configuration of the jurisdiction $I$ with its fitness value (real number).

The fitness value of a string is the sum of fitness contributions of its $\mathrm{N}$ elements. More formally we define the map $F_{i}$ as:

$$
F_{i}\left(x_{i 1}, x_{i 2}, \ldots x_{i N}\right)=\sum_{j=1}^{N} F_{i j}\left(x_{i 1}, x_{i 2}, \ldots x_{i N}\right)
$$

where $F_{i j}\left(x_{i l}, x_{i 2}, \ldots, x_{i N}\right)$ is the fitness contribution of the string element $x_{i j}$, given its configuration $\left(x_{i j}=0\right.$ or 1$)$.

$F_{i j}$ is treated as a random real number in a unit interval. The above notation is used to formalize the concept of interdependence, since the fitness contribution of $x_{i j}$ may depend not only on the configuration of this element, but also the configuration of the other elements of the string.

$K_{i j} \leq N-1$ is the number of string elements that are interdependent with respect to $x_{i j}$, so $K_{i j}+1$ is the number of the non-redundant argument of $F_{i j}\left(x_{i l}, x_{i 2}, \ldots, x_{i N}\right)$. For simplicity we assume that $K_{i j}$ is constant in all jurisdictions:

$K_{i j}=K=, j=1, \ldots, N \quad i=1, \ldots, G$

In the absence of interdependence $(K=0), F_{i j}\left(x_{i 1}, x_{i 2}, \ldots, x_{i N}\right)$ can be written as:

$$
F_{i j}\left(x_{i j}\right) \text { e } F_{i j}\left(X_{i 1}, X_{i 2}, \ldots . X_{i V}\right)=\sum_{j=1}^{N} F_{i j}\left(x_{i j}\right)
$$

The level of public expenditure with the highest fitness in $A_{i}$ is then identified by the string such that the 
configuration of each element $x_{i j}$ maximizes the fitness contribution $F_{i j}\left(x_{i j}\right)$ of that element. The fitness landscape of the Jurisdiction $i$ is the graph of $F_{i}$ on $A_{i}$. In a walk that combines $x_{i}$ to $x_{i}^{\prime}$ is a sequence of strings such that $x_{i}$ and $x_{i}^{\prime}$ are respectively the first and last element of the sequence, and the distance between each pair of adjacent elements of the sequence is $d=1$.

A walk that joining $x_{i}$ to $x_{i}^{\prime}$ and is minimal if the distance to $x_{i}^{\prime}$ is strictly decreasing on this "walk".

$x^{\prime}{ }_{i}$ is a local maximum of $F_{i}\left(x_{i 1}, x_{i 2}, \ldots, x_{i N}\right)$ on $A_{i}$ if and only if on every walk that joining $x_{i}^{\prime}$ to a string $y_{i}$ such that $F_{i}\left(y_{i}\right)>F_{i}\left(x_{i}^{\prime}\right)$ there is a $y_{i}^{\prime}$ such that $F_{i}\left(y_{i}^{\prime}\right)<F_{i}\left(x_{i}^{\prime}\right)$ e $d\left(x_{i}^{\prime}, y_{i}^{\prime}\right)<d\left(x_{i}^{\prime}, y i\right)$ (Note 8).

Suppose that $K=0$. If $x_{i}$ is a global maximum of $F_{i}$ on $A_{i}$, and $y_{i}$ is an arbitrary string in this set, then $F_{i}$ does not diminish in any shortest walk joining $x_{i}$ to $y_{i}$.

The proposition is self-evident. Because the walk is minimal, there must be many steps along the path as there are elements of $y_{i}$, which differ in their configuration, from the corresponding element of $x_{i}$. At every step along the path, the distance from $x_{i}$ decreases, since there is another element of $y_{i}$ that has the same value of the corresponding element of $x_{i}$. This value maximizes the fitness contribution of the element because $x_{i}$ is a global maximum, without reducing the fitness contribution of the other elements (Because $\mathrm{K}=0$ ) (Note 9). If $K=0$, the fitness landscape of the jurisdiction $i$ has at most one local optimum of $F_{i}$ on $A_{i}$ which corresponds to global optimum (Note 10).

We suppose $K>0$. The choice of configuration to maximize the fitness contribution of the element $x_{i j}$, given the configuration of the other $\mathrm{N}-\mathrm{l}$ elements of the string, cannot positively contribute to the general fitness level of public spending of jurisdiction $i$. The reason is that interdependence implies the possibility of a feed-back of uncertain sign stemming from the new configuration of the $x_{i j}$ to the fitness contribution of the other elements. This is equivalent to the possibility that there may be more local optima. The situation is illustrated in Figure 1 with reference to the simple case $N=2$ and $K=1$.

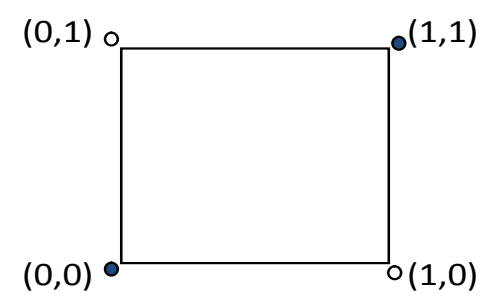

Figure 1. Landscape with $K>0$

In this example, the set $A_{i}$ of the possible levels of public spending in the jurisdiction is composed of 4 strings. Strings $(0.0)$ and (1.1) are the local optima. The path that joints the strings is done on the sides of a square but not on its diagonal because the diagonal steps involve simultaneous changes of many elements, not just one. By construction we know that in each path joining (0.0) to (1.1) the fitness function does not have a monotonic behavior.

Finally, we consider the greatest interdependence $(K=N-1)$. The fitness landscape is random in the sense that the fitness values of the neighbors are totally uncorrelated. A change (from 0 to 1 ), or vice versa in the configuration of a single element, say element $j$ of the level of public spending of the jurisdiction, not only assigns a new random fitness contribution to $F_{i j}$, but also a new random contribution $F_{h i}$ to each component of $h(h=1, \ldots, N$ (Note 11). The reason is that now $x_{j i}$ is not a redundant argument of $F_{h i}(h=1, N)$ (Note 12). The statements are based on the following assumptions.

Since $K=N-1$ the fitness values are not correlated; each string in a landscape has a probability $1 /(N+1)$ to be a local optimum and the expected value of local optima is $2^{N} /(N+1)$.

In each landscape the lower local optimum has a higher fitness value than the fitness value of the other $N$ strings. The fitness value of the local optimum can be understood as the maximum in a set of $2^{N}$ fitness values (Note 13).

On average, the higher $\mathrm{K}$ implies that: the higher is the number of local optima, the shorter is the minimal path that connects a random string in $A_{i}$ to the nearest local optimum; the lower the correlation between fitness values $F_{i}$ of neighboring strings $x_{i}, y_{i}$.

\subsection{The Role of Co-Evolution and Interdependences}

The choice to represent the co-evolution tends to emphasize that a change in the tax planning of a jurisdiction 
creates new and different opportunities or disadvantages for other jurisdictions. In other words, a movement of a jurisdiction along the fitness landscape can deform the fitness landscape of other jurisdictions. Here the fitness landscape of a jurisdiction is interpreted as the graph of a map that associates each possible variant of the state (configuration) of a jurisdiction with his fitness level, interpreted as a measure of its efficiency in a given environment and in a given time.

We consider pairs of levels of public spending in $G$ jurisdictions of the state. We also consider the hypothesis that a single level is evolving in each jurisdiction. Therefore, the efficiency of public spending here refers to the level of public spending of jurisdiction $i(i=1, \ldots, G)$. On any given landscape, the dynamics are assumed to be with reduced randomness, but, in line with the conclusions of the preceding paragraph, the same qualitative results are obtained when exploratory dynamics are considered totally random. The opportunity to optimize its tax system that is based on the interdependence between the elements, we remember, imply that, in general, the fitness of the $2^{N}$ states of $i$, depends on the current state of the other $G-1$ jurisdictions. Following Kauffman (1993), we can predict these effects such as deformities of the fitness landscape of the jurisdiction $i$, triggered by changes in the other $G-1$ jurisdictions. More precisely, we consider the changes in fitness levels of public spending in the jurisdiction $i(i=1, \ldots, G)$. The changes in the landscape can be global or local. If the relationships of interdependence between jurisdictions are limited to small segments of the string, the change of a single element does not induce a change in the global fitness landscape of another jurisdiction. However, because the State is composed of many jurisdictions, a multiplicity of individual change takes place simultaneously. Hence $G$ is larger than $N$, the greater the probability of a global change of the landscape. If $G$ is very small relative to $N$, the case of deformation bases on the assumption that global interdependence across jurisdictions is pervasive. Situations of complete interdependence are defined by the fact that each component of each string is connected to every other component of every other string. A single change in a state of an element is therefore sufficient to set up an entirely new landscape for any other jurisdiction. We use this rather extreme hypothesis, because it suggests an approach that strongly takes into account the co-evolution, whence the general qualitative effects of complementarity are more easily detected. So we define $C$ the number of co-evolving systems.

The economic dynamics of $G$ jurisdictions are determined by their interdependences, and the following tables describe the list of fitness values of each element corresponding to each state of level of public spending in the remaining jurisdictions.

The first element of the list is the one with the highest fitness value. The possibility that adjacent elements in the list have the same fitness value is excluded, because the event could be an irrelevant fluke.

Two examples are shown below for the case: $N=2, C=2$. The two jurisdictions are called $\alpha$ and $\beta$, and, by way of example, $\alpha 00$ is the state of public expenditure $(0,0)$ of jurisdiction $\alpha$. Table 1 refers to the case $K=0$, Table 2 to case $K=1$.

Table 1. Interdependences with $\mathrm{K}=0$

\begin{tabular}{llllllllll}
\hline $\operatorname{Se} \beta_{00}:$ & $\alpha_{10}$ & $\alpha_{11}$ & $\alpha_{01}$ & $\alpha_{00}$ & Se $\alpha_{00}:$ & $\beta_{11}$ & $\beta_{10}$ & $\beta_{01}$ \\
$\operatorname{Se} \beta_{01}:$ & $\alpha_{11}$ & $\alpha_{10}$ & $\alpha_{01}$ & $\alpha_{00}$ & $\operatorname{Se} \alpha_{01}:$ & $\beta_{10}$ & $\beta_{00}$ & $\beta_{01}$ & $\beta_{11}$ \\
$\operatorname{Se} \beta_{10}:$ & $\alpha_{01}$ & $\alpha_{00}$ & $\alpha_{11}$ & $\alpha_{10}$ & $\operatorname{Se} \alpha_{10}:$ & $\beta_{00}$ & $\beta_{10}$ & $\beta_{11}$ \\
$\operatorname{Se} \beta_{11}:$ & $\alpha_{00}$ & $\alpha_{10}$ & $\alpha_{01}$ & $\alpha_{11}$ & $\operatorname{Se} \alpha_{11}:$ & $\beta_{01}$ & $\beta_{11}$ & $\beta_{00}$ & $\beta_{10}$ \\
\hline
\end{tabular}

Table 2. Interdependences with $\mathrm{K}=1$

\begin{tabular}{llllllllll}
\hline Se $\alpha_{00}:$ & $\beta_{00}$ & $\beta_{11}$ & $\beta_{01}$ & $\beta_{10}$ & Se $\beta_{00}:$ & $\alpha_{00}$ & $\alpha_{11}$ & $\alpha_{01}$ \\
Se $\alpha_{01}:$ & $\beta_{00}$ & $\beta_{11}$ & $\beta_{10}$ & $\beta_{01}$ & Se $\beta_{01}:$ & $\alpha_{00}$ & $\alpha_{11}$ & $\alpha_{01}$ & $\alpha_{10}$ \\
Se $\alpha_{10}:$ & $\beta_{11}$ & $\beta_{00}$ & $\beta_{10}$ & $\beta_{01}$ & $\operatorname{Se} \beta_{10}:$ & $\alpha_{11}$ & $\alpha_{00}$ & $\alpha_{10}$ \\
$\operatorname{Se} \alpha_{11}:$ & $\beta_{11}$ & $\beta_{00}$ & $\beta_{01}$ & $\beta_{10}$ & $\operatorname{Se} \beta_{11}:$ & $\alpha_{11}$ & $\alpha_{00}$ & $\alpha_{01}$ & $\alpha_{10}$ \\
\hline
\end{tabular}

The time is discrete, and at each $t$ time each configuration moves from the present state to fittest neighbor through simultaneous changes. The representation space of the dynamics induced by a given interdependence pattern between $G$ jurisdictions, given the co-evolution, is the hypercube $\{0.1\} N G$. Each hyper-row, or hyper-column of this representation space consists of an ordered series of $2^{N}$ elements, where each element, or a point $\left(x_{1}, \ldots, x_{G}\right)$ is an ordered list of tax configurations (Note 14)one for each jurisdiction. 
The neighbor of a point in state space is an ordered list $\left(y_{l}, \ldots, y_{g}\right)$ such that each $y_{i}$ is a string of $N$ binary codes $N$ and $d\left(x_{i}, y_{i}\right)<1$.

A point in the state space has $N G$ neighbors. Each element of a hyper-row (or hyper-column) is therefore a configuration of a jurisdiction, and moves on the same hyper-line (or hyper-column) on which we meet all the possible states of the jurisdiction $i$, while the state of other $G-1$ jurisdiction is unchanged.

Recall that for $K=0$ each fitness landscape has one peak and that, by construction, each hyper-row (or hyper-column) refers to the fitness landscape of a given jurisdiction. Suppose that the level of interdependence is given.

A rest point in the state space corresponding to this model is that all jurisdictions are simultaneously on a peak of fitness. If and only if $K=0$, on every hyper-row (or hyper-column) in the state space there is at most one rest point in which the co-evolution slows down as shown in Figures 2 and 3. Figure 2 shows in fact, associated dynamics in the representation space of possible solutions $\{0,1\} 4$ determined by the model of interdependence indicated in Table 2.

$$
\begin{array}{lll}
\left(\alpha_{01}, \beta_{01}\right) & \left(\alpha_{01}, \beta_{10}\right) \longmapsto\left(\alpha_{11}, \beta_{00}\right) & \left(\alpha_{10}, \beta_{01}\right) \longmapsto\left(\alpha_{00}, \beta_{11}\right) \\
\left(\alpha_{00}, \beta_{01}\right) & \longrightarrow\left(\alpha_{00}, \beta_{00}\right) & \left(\alpha_{10}, \beta_{01}\right) \\
\left(\alpha_{01}, \beta_{00}\right) & \left(\alpha_{11}, \beta_{10}\right) \\
\left(\alpha_{10}, \beta_{00}\right) & \longrightarrow\left(\alpha_{11}, \beta_{11}\right) \\
\left(\alpha_{00}, \beta_{10}\right) & \left(\alpha_{01}, \beta_{11}\right) \\
& \left(\alpha_{11}, \beta_{01}\right)
\end{array}
$$

Figure 2. Possible solutions when $\mathrm{K}>0$

When $K>0$, when the co-evolution begins to decrease, not all jurisdictions are necessarily on a global optimum of their landscape (see, for example, the state $\left(\alpha_{11}, \beta_{00}\right)$ of Fig 2 . Some may be at the global peak while some others at a strictly local peak, or they may be simultaneously at a strictly local peak.

The number of admissible patterns of interdependence depends on the parameters $N$ and $K$ and the co-evolution. Since there are $2^{\mathrm{N}}$ different states of a given jurisdiction, there are $2^{\mathrm{N}}$ ! re-ordering of these different strings based on their fitness value.

When $K=N-1$, each of these re-ordering is admissible. However, if $K=0$, two adjacent strings differ on every admissible re-ordering in one and only one element.

Since every configuration to the jurisdiction $i(i=1, \ldots, G)$ can be coupled with $2^{\mathrm{N}(\mathrm{G}-1)}$ different states of the remaining jurisdictions, we obtain $\left[\left(2^{\mathrm{N}} !\right) 2^{\mathrm{N}(\mathrm{G}-1)}\right]^{\mathrm{S}}$ possible patterns of interdependence for the case $\mathrm{K}=\mathrm{N}-1$, where the parameter $S$ identifies the degree of co-evolution, and a considerably lower number of possibilities for $K=0$.

Any admissible model gives rise to an evolutionary dynamics in phase space, which is a set of $2^{\mathrm{NS}}$ trajectories, each starting from a different initial condition in phase space (see Fig 2 and 3).

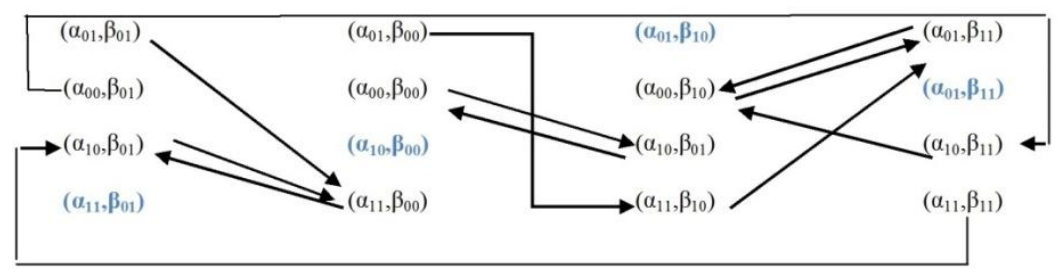

Figure 3. Stationary points of co-evolution

\subsection{Constraints in Random Model: What Change in Landscape Exploration}

To allow a complex system to move in the landscape by dividing it into several pieces can clarify the problems within the system that, oversized, has difficult to explore the entire territory, to design and to test new evolutionary paths. 
To divide the State in more local units of government cannot just give the entire tax system the needed degree of flexibility to adapt to the socio-economic changing, but also to find and to exploit all facets of the local microcosm. For happening this it is essential that the size of local jurisdictions is right. We saw earlier how Buchanan has resolved the matter.

For example, to check what the right size is, we can use the fitness landscape in the following way. In the contemporary States the levels of government unlikely exceed the number of three: the central one, the middle (regions, cantons) and local (municipalities, provinces). Each level corresponds to a different dimension, taking into consideration economic and political considerations. On the intermediate, often in conflict with the central level, most often it is the focus of the system of territorial government. Consequently, the local units have very few skills.

From an economic point of view it is important to take into account also the size of the externality effects, the preferences of citizens, administrative costs and economies of scale. These constraints are also added to those brought as dowry by patching: the patches should be neither too large, otherwise the complex system is likely to crystallize in a single configuration and hang in an area of the landscape, nor too small, if it doesn't want that the pure chaos reigns supreme.

These new restrictions are necessary to ensure to the financial structure of a country an appropriate process for future development, aimed at achieving the goals of economic theory of fiscal federalism.

Let's see how a jurisdiction can precede the exploration in the fitness landscape. Here we compare the properties of Kauffman's random exploration with a dynamic that reduces the randomness by introducing small constraints to be respected in the choice of fitness contributions. The choice rises by the assumption that the introduction in a totally random model, of some qualitatively and quantitatively important information, without falling into over-simplistic, increases the effectiveness of the use of complex tools. At each time $t$, the jurisdiction $i$ doesn't have a perfect knowledge of $A_{i}$, because the perception of a potentially profitable combination of elements $x_{i} \in A_{i}$, and even more, the information on its fitness $F\left(x_{i}\right)$, is available only if $x_{i}$ is in the neighborhood of the string that defines the tax configuration of jurisdiction $i$ at time $t$. The information, even when it can be codified, does not immediately translate into knowledge that can be exploited for useful purposes. The transformation of information into knowledge requires understanding, learning and adaptation.

We can assume that this not encode information can be gained through experience. Unlike sectors where every change is always associated with a random mechanism, here we try to know how the research can proceed through the combination of random explorations and more targeted explorations aimed to achieve pre-selected goals. According to the dynamic of $N K$ model induced by random exploration on a fitness landscape the neighbor element $x_{i}^{\prime}$ of the current state $x_{i}$ is randomly selected at any time (Note 15). The fitness value $F\left(x_{i}{ }_{i}\right)$ was then examined, and a movement toward $x_{i}^{\prime}$ occurs if $F\left(x_{i}{ }_{i}\right)>F\left(x_{i}\right)$.

A greater focus on the intentional components of research generates the assumption that at any moment a system moves one step from pre-determined state to the state identified by the string with the highest fitness value in the given neighborhood.

This modeling strategy produces a slightly different dynamic on $A_{i}$. This comes out when the sequence of a neighbor $x_{i}$ of $x^{\prime}{ }_{i}$ uses combinations of intentional and random choices: in each time $n<N$ components of $x_{i}$ with relatively low contributions to fitness are intentionally selected, one of which is randomly selected and its configuration modified. As before, a move towards $x_{i}^{\prime}$ occurs if $F\left(x_{i}^{\prime}\right)>F\left(x_{i}\right)$. The figure shows the dynamics of a single jurisdiction on the fitness landscape $N=2$ and $K=1$ described in Fig. 1. Black points identify the local peaks of the fitness landscape. The string (1.1) is the global optimum $\mathrm{F}_{\mathrm{i}}$ on $\mathrm{A}_{\mathrm{i}}$. The string (0.0) is a degenerate basin of attraction, which coincides with the string itself.

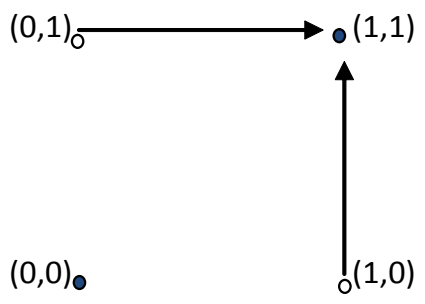

Figure 4. Robust landscape 
Figure 4 clarifies how the landscape in this example is completely robust in the sense that each neighbor of a string that is not a local optimum is a local isolated peak.

It may be instructive to compare the asymptotic average properties of NK model of Kauffman's random exploration and the dynamics of the model with bounded randomness on fitness landscape randomly generated for extreme cases of absence and complete interdependence. The main differences are: when $K=0$, the full randomly exploration and the bounded randomness exploration at the end reach both the global optimum of the landscape, the number of steps required is smaller in the bounded randomness, because every step is taken towards the pre-selected direction (Note 16)

$0<K<N-1$ the dynamics of randomly exploration converges to the average of global optimum of the landscape. The average fitness value $F^{*}(N, K)$ of a local optimum changes with $N$ and $K$.

For finite values $N$, the asymptotic deterministic dynamic on a landscape $0<K<N-1$ climbs a local optimum of fitness, which is surely above average. If $K=N-1$, the fitness of highest optimum at average drops to 0.5 when $\mathrm{N}$ tend to infinity. The same is not true if $N$ grows to infinity, but $K$ remains constant.

\section{Conclusions}

Having shown that fiscal federalism is to be understood as a dense network of economic-financial relationships between different coevolving complex and adaptive systems (the central and local government), linked by strong interdependencies, it clarifies even more the goal of the paper aimed to study fiscal federalism from a dynamic and evolutionary point of view, seeking solutions to problems posed by traditional economic theory with new analysis tools of Complexity.

The solution of a problem built on an adaptive complex system, cannot be searched as if we were solving a simple problem without interconnections. To identify in fact, a single optimal solution while it is possible for a simple system, it is not for a complex system.

In this case it is only possible on the basis of its numerous connections, to determine the process by which different solutions may emerge, more or less favorable to the resolution of the problem. Taking into account the existence of multiple solutions, the same research can be done through a searching algorithm on a fitness landscape, a dynamic landscape in which complex adaptive systems are moving in search of optimum conditions. The configuration of this landscape is strongly conditioned by the presence of co-evolution and interdependencies. Also the jurisdictions as result of fiscal decentralization can be regarded as evolving complex systems, although smaller. From these assumptions and on the basis of evolutionary dynamics, we analyzed the behavior of jurisdictions to develop a model to identify their optimal fiscal configurations by using $N K$-model.

The $N K$-model can be considered as composed of two distinct components: a specific problem and a searching algorithm in the space of possible solutions. As we have said, the problem is a set of possible solutions represented as binary strings, each associated with a fitness value, which is in fact the pay-off of that solution. The searching algorithm consists of repeated mechanism in order to scan the solution space from a (usually randomly chosen) initial string, or a binary $\mathrm{N}$-dimensional space. The ongoing research is defined in terms of rules on how to move from one point to another. For example, the typical search, originally proposed (Kauffman, 1993) is to choose randomly a string in which changing one bit, in the case that the changed string has fitness higher than the current one, the new string is accepted, and otherwise it is rejected.

The repeated application of the algorithm generates a pattern in the space of possible solutions. The pattern ends when the rule reaches a string from which all possible strings within the space of solutions were rejected. Two aspects make particularly attractive the $N K$-model. First, you can determine the whole must be the solution space, or the fitness landscape. Building a landscape with few or no interactions (represented by the value of $K$ ) means to generate the equivalent of simple problems, increasing $K$ generates a complex problem.

The second aspect is the representation of the $N K$-model searching algorithm. The $N K$-model assumes a local search. Local, because the research involves the inability to observe the space beyond the near immediate focusing on the goal of improving their present condition. The two aspects, complexity through interaction and local search, paradoxically leads to a simplified and then manageable of many real situations.

It's an interesting tool because it provides the opportunity for the researcher to represent and control the two aspects of problem solving: the complexity of the problem and the degree of expertise for finding a solution. It' $s$ possible to use NK model to generate and to evaluate the space formed by the two dimensions of the complexity of the problems and skills in the resolution strategy in order to represent both aspects of a real-world in small-scale. The use of the $N K$-model arises from the possibility of establishing a sort of relationship between the skills of decision makers and the related difficulty of intervention in economic policy. In this case, it is no 
more relevant that the modeled problem is much simpler than the real one, since even the solution strategies are modeled in a much less sophisticated way. By controlling both aspects we can be expected that the properties of the set that includes the solutions generated in the model are similar to the set of real solutions generated in real systems with an equivalent ratio of task and skills difficulty in finding a solution.

Moreover, if Kauffman's work exploits to a large extent the simulation of large complex systems, we pursue an analytical approach to the presentation of the main qualitative properties of the models. Starting with Kauffman's work, we built a model by introducing some constraints to the full causality. We compared then the properties of Kauffman's random exploration with a dynamic that reduces the randomness by introducing small constraints to be respected in the choice of fitness contributions. The choice rises by the assumption that the introduction in a totally random model, of some qualitatively and quantitatively important information, without falling into over-simplistic, increases the effectiveness of the use of complex tools.

In this scenario, interdependence constraints have played a selective role influencing the adaptability of systems. In this way, the interdependence constraints have contributed to reduce the uncertainty and disorder in a system, considered as a set of evolving complex systems.

Moreover, according to Kauffman' result, the model shows the complex nature of each system, justified by its ability to associate order and disorder. The disorder is represented by the random choice of fitness contributions fitness, order, however, by a coherent structure, produced by the model and made of configurations, adaptive walk, fitness landscape and fitness value.

To take a systematic relationship between interaction and fitness contributions means that each fitness landscape is drawn from a distribution such that the degree of interaction of an element is correlated with its fitness contribution. A stronger interaction leads to stronger constraints of complementarity. On this premise, the more closely integrated fitness landscape is even more rugged in the average. Moreover, as evidenced by the results of Kauffman, in a landscape more rugged local optima are more numerous, although their average fitness value may be lower. In addition, walks to the local optima involve fewer steps. These properties were used to demonstrate that, at every stage of a co-evolutionary process, the systems evolving on a rugged landscape are more likely to be simultaneously on a peak of fitness, and then to move towards a local optimum. If the systems have a sufficiently large number of $\mathrm{N}$ elements, there is a trade-off between the probability that a process co-evolve towards a stable local peak and the average fitness value of a peak. Thus, it confirms that systems with an intermediate degree of interaction have a selective advantage against competitors, characterized by very high or very low complementarity constraints.

These properties are always true, no matter if evolution proceeds by random exploration of such trial and error (as most assumed by Kauffman), or by choosing to impose constraints, as in the proposed model, to help identify optimal choices within a set of local choices. Once the systems are simultaneously on a peak of fitness, co-evolution tends to decrease.

Finally it stressed that the decision to introduce constraints to randomness rises from on the assumption that the introduction, in a totally random model, of some qualitatively and quantitatively important information, without falling into over-simplifications, increases the effectiveness of using of complexity tools.

\section{References}

Ahmad, E., \& Brosio, G. (2006). Handbook of Fiscal Federalism. Cheltenham, UK: Edward Eldgar. https://doi.org/10.4337/9781847201515

Arthur, W. B., Durlauf, S. N., \& Lane, D. (1997). The Economy as an Evolving Complex System II. Santa Fe Institute Studies in the Sciences of Complexity, 27. Addison-Wesley, Reading, Massachusetts.

Barabasi, A. L. (2003). Linked: How Everything is Connected to Everything Else and What it Means for Business, Science, and Everyday life. New York: Plume.

Barkley, R. J. (2012). Complexities of Natural Selection Dynamics, Global Analysis of Dynamic Models. Economics and Finance, 429-442. https://doi.org/10.1007/978-3-642-29503-4_16

Barkley, R. J. Jr. (1999). Book review: The economy as an evolving complex system II. In W. B. Arthur, S. N. Durlauf, \& D. A. Lane (Eds.), Discrete Dynamics in Nature and Society (Vol. 3, No. 1 pp. 71-74). https://doi.org/10.1155/s1026022699000114

Bertuglia, C. S., \& Vaio, F. (2006). Nonlinearity, Chaos and Complexity. The Dynamics of Natural and Social Systems. Choice Reviews Online, 43(5), 43-2763. https://doi.org/10.5860/choice.43-2763

Besley, T., \& Coate, S. (2003). Centralized versus decentralized provision of local public goods: A political 
economy approach. Journal of Public Economics, $2611-2637$. https://doi.org/10.1016/S0047-2727(02)00141-X

Brennan, G., Buchanan, J. M., \& Prest, A. R. (1981). The Power to Tax: Analytic Foundations of a Fiscal Constitution. Economica, 48(192), 1-14. https://doi.org/10.2307/2553704

Buchanan, J. M. (1965). An Economic Theory of Clubs. Economica, 32(125), 1-14. https://doi.org/10.2307/2552442

Carminati, M. (1999). Complementarity, Opportunity and the Co-Evolution of Technique. Quaderni del Dipartimento di Politica Economica, 260. Università di Siena.

Colander, D., Holt, R. P. F., \& Rosser, J. B. (2004). The Changing Face of Mainstream Economics. Review of Political Economy, 16(4), 485-499. https://doi.org/10.1080/0953825042000256702

Frenken, K. (2001). Modelling the organisation of innovative activity using the NK model. Retrieved from http://www.druid.dk/conferences/nw/paper1/frenken.pdf

Frenken, K. (2005). Innovation, Evolution and Complexity Theory (Cheltenham \& Northampton MA: Edward Elgar).

Frenken, K. (2006) Technological innovation and complexity theory. Economics of Innovation and New Technology, 15(2), 137-155. https://doi.org/10.1080/10438590500141453

Frenken, K., \& Valente, M. (2004). A fitness landscape approach to decentralised problem-solving. Utrecht University.

Gerrits, L. M., \& Marks, P. K. (2014). The evolution of Wright's (1932) adaptive field to contemporary interpretations and uses of fitness landscapes in the social sciences. Biology and Philosophy, 30(4), 459-479. https://doi.org/10.1007/s105390149450-2

Kauffman, S. A. (1993). The Origin of Order: Self-Organization and selection in Evolution. Oxford University Press. USA.

Kauffman, S. A. (1995). At Home in the Universe: The Search for Laws of Self-Organization and Complexity. New York, Oxford University Press.

Levinthal, D. A. (1997). Adaptation on rugged landscapes. Management Science, 43, $934-950$. https://doi.org/10.1287/mnsc.43.7.934

Lookwood, B. (2007). The Political Economy of Decentralization. In E. Ahmad, \& G. Brosio (Eds.), Handbook of Fiscal Federalism, 33-60. Cheltenham, U.K.: Edward Elgar. https://doi.org/10.4337/9781847201515.00008

Musgrave, R. A. (1959). The theory of public finance: A Study in Public Economy. New York, Mcgraw-Hill Book.

Nelson, R. R., Winter, S. G., \& Loasby, J. B. (1983). An Evolutionary Theory of Economic. The Economic Journal, 93(371), 652. https://doi.org/10.2307\%2F2232409.

Oates, W. E. (1972). Fiscal federalism. New York: Harcourt Brace Jovanovich.

Oates, W. E. (1999). An Essay on Fiscal federalism. Journal of Economic Literature, 37(3), 1120-1149. https://doi.org/10.1257/jel.37.3.1120

Oates, W. E. (2005). Toward a Second-Generation Theory of Fiscal Federalism. International Tax and Public Finance, 12(4), 349-373. https://doi.org/10.1007/s10797-005-1619-9.

Oates, W. E. (2008). On the Evolution of Fiscal federalism: Theory and Institution. National Tax Journal, 61(2), 313-322. https://doi.org/10.17310/ntj.2008.2.08

Oliver, D., \& Roos, J. (1999). Striking a balance: Complexity and knowledge landscapes. London, McGraw-Hill Publishing Company.

Pagano, U. (2013). The origin of organizational Species. In A. Nicita, \& U. Pagano (Eds.), The Evolution of Economic Diversity, 21-47. Routledge Taylor \& Francis Group.

Post, D. G., \& Johnson, D. R. (1998). Towards a new theory of decentralized decision-making in complex systems. Chicago-Kent Law Review.

Seabright, P. (1996) Accountability and decentralization in government: An incomplete contracts model. European Economic Review, 40, 61-89. https://doi.org/10.1016/0014-2921(95)00055-0 
Spahn, P. B. (2006). Contract Federalism. In E. Ahmad, \& G. Brosio (Eds.), Handbook of Fiscal Federalism (pp. 182-199). Cheltenham, U.K.: Edward Elgar. https://doi.org/10.4337/9781847201515.00014

Stacey, R. D. (1995). The science of complexity: An alternative perspective for strategic change processes. Strategic Management Journal, 16, 477-495. https://doi.org/10.1002/smj.4250160606

Stacey, R. D. (2003). Strategic Management and Organizational Dynamics. The challenge of complexity. London UK: Prentice Hall.

Tiebout, C. M. (1956). A pure Theory of Local Government Expenditure. Journal of Political Economy, 64(5), 416-424. https://doi.org/10.1086/257839

Weingast, B. R. (1995). The Economic Role of Political Institution: Market Preserving Federalism and Economic Development. The Journal of Law, Economics, and Organization, 11(1), 1-31. https://doi.org/10.1093/oxfordjournals.jleo.a036861

Westhoff, F. H., Yarbrough, B. V., \& Yarbrough, R. M. (1996). Complexity, Organization, and Stuart Kauffman's The Origins of Order. Journal of Economic Behavior and Organization, 29(1), 1-25. Elsevier. https://doi.org/10.1016/0167-2681(95)00049-6

Wright, S. (1932). The roles of mutation, inbreeding, crossbreeding, and selection in evolution. Procedeengs of.the Sixth. International. Congress. of Genetic, 1, 356-366.

\section{Notes}

Note 1. For a review of the latest literature on fiscal relations between different levels of government see Handbook of Fiscal Federalism by Ahmad E. and Brosio G. (2006).

Note 2. Complementarity in this case means that the elements must be used and to act together in order to maximize the degree of fitness of the system to which they belong.

Note 3. In a system with no spillover effects, each element's fitness contribution is a function only of its own state (Post \& Johnson, 1998, p. 1075).

Note 4. In a system with spillover effects, "an element's 'spillover set' consists of those elements whose fitness contribution is a function of that element's state" (Post \& Johnson, 1998, p. 1079).

Note 5. A detailed explanation about the algorithms operation is in Post and Johnson (1999).

Note 6. See the original model in Carminati (1999). Here we adapt the method to the aim of the paper.

Note 7. We therefore define two or more neighbouring combinations that differ for a single element: $d=1$.

Note 8 . With reference to the case $K=0$, it is important to remember how a change from 0 to 1 , or vice versa, in the configuration of a single string element, does not affect the fitness contribution of the other components.

Note 9 . The probability that a randomly chosen string in a landscape $K=0$ is a local peak is $1 / 2^{N}$. Let $F^{*}(N, K)$ be the expected fitness of a local peak. $F^{*}(N, 0)$ is independent of $N$ and can be expressed as $E\left[\sum_{j=1}^{N} \operatorname{Max}\left(a_{j}, b_{j}\right)\right]=0.666$

where $\left(a_{j}, b_{j}\right)$ are $\mathrm{N}$ couples of real random numbers uniformly distributed on the unit interval.

Note 10. This is easily demonstrable by supposing the contrary. If $x_{i}$ is a maximum of $F_{i}$ on $A_{i}$ There may be in the same space an isolated maximum (local or global), $y_{i} \neq x_{i}$ of the fitness function $F_{i}$. By construction $F_{i}$ has a non-monotonic behavior on every minimal path joining $y_{i}$ and $x_{i}$.

Note 11. Footnote 9 implies that $F^{*}(1,0)=0.666$. If $N>1 F^{*}(N, N-1)$ first grows above 0.666 and then decreases monotocally to 0.5 . Moreover if $K=N-1$ then $F^{*}(m N, K)=F^{*}(N, K)$ for any $m \geq 1$. This suggests that $F^{*}(N, K)$ remains approximately constant as $\mathrm{N}$ grows to infinity, while $K$ is fixed at $N-1$.

Note 12. The fitness value of each element on a landscape $K=N-1$ is a random number, uniformly distributed between 0,1 . The probability that a randomly chosen element of the landscape is a local peak (its fitness value is higher than its $N$ neighbours) is $1 /(N+1)$. Then there are on average $2^{N} /(N+1)$ local peak on a landscape $K=$ $N-1$.

Note 13. This involves lower and upper bounds to $\mathrm{F}^{*}(\mathrm{~N}, \mathrm{~N}-1)$ : $E\left[\operatorname{Max}\left(\alpha_{1}, \alpha_{2}, \ldots ., \alpha_{m}\right)\right]<F *(N, N-1)<E\left[\operatorname{Max}\left(\beta_{1}, \beta_{2}, \ldots, \beta_{m}\right)\right]$.

Where each $\alpha_{\mathrm{m}}$ e $\beta_{\mathrm{m}}$ i san average of $\mathrm{N}$ random numbers in the unit interval $\mathrm{m}=\mathrm{N}+1, \mathrm{M}=2^{\mathrm{N} .}$. Since the expected 
fitness value of intermediate local optima uniformly distributed between the lower and upper bounds above, we have: $F *(N, N-1)=\left\{E\left[\operatorname{Max}\left(a_{1}, a_{2}, \ldots, a_{m}\right)\right]+E\left[\operatorname{Max}\left(b_{1}, b_{2}, \ldots, b_{M}\right)\right]\right\} / 2$

Order statistic shows that for $\left\{E\left[\operatorname{Max}\left(\alpha_{1}, \alpha_{2}, \ldots, \alpha_{m}\right)\right]+E\left[\operatorname{Max}\left(\beta_{1}, \beta_{2}, \ldots, \beta_{M}\right)\right]\right\} / 2 \approx 0.7 \quad 4 \leq N \leq 10$.

Consequently $\mathrm{F}^{*}(\mathrm{~N}, \mathrm{~N}-1)$ decreases as $\mathrm{N}$ increases and converges to 0.5 as $\mathrm{N}$ grows to infinity because each single sample average $\alpha_{\mathrm{m}}$ and $\beta_{\mathrm{m}}$ must behave accordingly. Moreover, we consider $K=N-1$ and $F^{*}(N, K)$ where $N=m N$ and $K=K$. Through a possible re-ordering of elements, every string of length $\mathrm{N}$ can be thought of as being composed of $\mathrm{m}$ segments of $\mathrm{N}$ elements each. Within each segment each element is connected to the $\mathrm{K}$ other elements. Thus, the fitness contribution of each component depends on its configuration $(0$ or 1$)$ and on the configuration of every other component of the same segment. Hence the expected fitness value of each segment is an average of $\mathrm{N}$ random numbers in the unit interval and is identical to the expected fitness contribution of every other segments. This holds independently of the size of $\mathrm{m}$.

Note 14. Strings of N binary codes.

Note 15. This amounts to a random selection of one element of xi and a change of its configuration (from 0 to 1 or vice versa).

Note 16 . The average fitness value $F^{*}(N, 0)$ of a global optimum is 0,666 .

\section{Copyrights}

Copyright for this article is retained by the author(s), with first publication rights granted to the journal.

This is an open-access article distributed under the terms and conditions of the Creative Commons Attribution license (http://creativecommons.org/licenses/by/4.0/). 